There were no neuropsychological correlates of specific psychiatric symptoms in this study, and indeed this seems to be a common negative finding in schizophrenia research. While only $67 \%$ of schizophrenic subjects demonstrated impaired WCST performance, using cut-offs generated from this study, it is to be remembered that WCST performance is not the only measure of pre-frontal functioning. The pre-frontal cortex is a large area of the brain!

Hunter Hospital

RODNEY MORICE

Newcastle, NSW

Australia

\section{Computerised tomography in schizophrenia}

SIR: In the article entitled "Computerised tomography in schizophrenia, 15 years on" by Lewis (Journal, 1990, 157 (suppl. 9), 16-24) there is one statement that I feel is unnecessarily dogmatic and probably wrong. Lewis states that "the degree of ventricular enlargement is convincingly not a function of how long a patient has been ill". In a recent review of this issue (Miller, 1989) over 30 studies were considered which contained data on the statistical relationship between abnormalities of CT findings in schizophrenia and age, duration of illness, duration of hospital stay, or number of episodes. The majority of these studies found no relationship with duration of illness, but a few did show increased abnormalities in those patients who had been ill longer. The latter group of papers generally studied younger patients with shorter durations of illness.

Ventricular enlargement occurs to some extent with increasing age in normal persons, and this correlation might explain those cases where CT abnormalities in schizophrenia increase in magnitude with increasing duration of illness. However, in two studies, the appropriate statistical analyses were performed to show that duration of illness does have a correlation with degree of CT abnormality, independent of the age correlation.

More recent scanning studies have supported the suggestion that, at least in the first few years of a schizophrenic illness, there is a progressive increase in brain abnormalities. For instance, a one to four and a half year CT follow-up study by Woods et al (1990) showed progressive enlargement between the two scans. Of all follow-up scanning studies carried out to date, this study used the youngest, and probably the least chronic patients. DeLisi et al (1991) carried out a NMR study of very early-stage schizophrenic patients and found significant correlation between some measures of loss of brain substance and duration of illness. Thus, in contrast to the statement by Lewis, this issue is by no means settled.

In my own article, I suggested that there is progressive loss of brain substance during the manifest illness, which occurs mainly in the first few years of the illness, particularly when there are episodes of unchecked psychosis. This hypothesis could be of considerable significance, if correct. It may mean that, once a diagnosis has been made, very careful attention to antipsychotic medication to prevent, as far as possible, all further episodes of psychosis, can to some extent prevent progressive brain cell loss, and perhaps also the tendency towards progressive decline in mental function over the years of the illness.

\section{University of Otago}

ROBERT MILLER

\section{Dunedin}

New Zealand

\section{References}

MILLER, R. (1989) Schizophrenia as a progressive disorder: relations to EEG, CT, neuropathological and other evidence. Progress in Neurobiology, 33, 17-44.

WoOds, B. T., Yurgelun-TOdD, D., Benes, F. M., et al (1990) Progressive ventricular enlargement in schizophrenia: comparison to bipolar affective disorder and correlation with clinical course. Biological Psychiatry, 27, 341-352.

DeLisı, L. E., Hoff, A. L., Schwartz, J. E., el al (1991) Brain morphology in first-episode schizophrenic-like psychotic patients: a quantitative magnetic resonance imaging study. Biological Psychiatry, 29, 159-175.

\section{Tardive dyskinesia and HLA}

SIR: I read with interest Brown \& White's report (Journal, February 1991, 158, 270-272) of their study on the possible association between tardive dyskinesia (TD) and the human leucocyte antigen (HLA), B44. However, I do not think that the methods and results reported either replicate or extend Canoso et als (1986) work, their stated aim, or support their conclusion that HLA B44 is not useful as a predictor of neuroleptic-induced TD.

Canoso et als 66 patients were all male, and no indication is given of their ethnic origin. Drs Brown \& White included both male and female subjects, and although of a "similar ethnic origin", their exact ethnicity is not stated. It is very unlikely that $\mathbf{4 0}$ long-term patients of a Scottish psychiatric hospital could be reasonably compared with 66 patients at a Massachusetts Veterans Administration facility for something as ethnically dependent as HLA haplotype. 